\title{
Optical two-beam trap in a polymer microfluidic chip
}

Palanco, Marta Espina; Catak, Darmin; Marie, Rodolphe ; Matteucci, Marco; Bilenberg, Brian; Kristensen, Anders; Berg-Sørensen, Kirstine

\section{Published in:}

Proceedings of SPIE 9922, Optical Trapping and Optical Micromanipulation XIII

Link to article, DOI:

$10.1117 / 12.2236465$

Publication date:

2016

Document Version

Publisher's PDF, also known as Version of record

Link back to DTU Orbit

Citation (APA):

Palanco, M. E., Catak, D., Marie, R., Matteucci, M., Bilenberg, B., Kristensen, A., \& Berg-Sørensen, K. (2016). Optical two-beam trap in a polymer microfluidic chip. In Proceedings of SPIE 9922, Optical Trapping and Optical Micromanipulation XIII (Vol. 9922). SPIE - International Society for Optical Engineering. Proceedings of SPIE The International Society for Optical Engineering https://doi.org/10.1117/12.2236465

\section{General rights}

Copyright and moral rights for the publications made accessible in the public portal are retained by the authors and/or other copyright owners and it is a condition of accessing publications that users recognise and abide by the legal requirements associated with these rights.

- Users may download and print one copy of any publication from the public portal for the purpose of private study or research.

- You may not further distribute the material or use it for any profit-making activity or commercial gain

- You may freely distribute the URL identifying the publication in the public portal 


\title{
Optical two-beam trap in a polymer microfluidic chip
}

\author{
Marta Espina Palanco ${ }^{\mathrm{a}}$, Darmin Catak ${ }^{\mathrm{b}}$, Rodolphe Marie $^{\mathrm{b}}$, Marco Matteucci $^{\mathrm{b}}$, Brian \\ Bilenberg $^{c}$, Anders Kristensen ${ }^{\mathrm{b}}$, Kirstine Berg-Sørensen ${ }^{\mathrm{a},{ }^{*}}$ \\ ${ }^{a}$ DTU Physics, Technical University of Denmark, 2800 Kgs Lyngby, Denmark \\ ${ }^{\mathrm{b}}$ DTU Nanotech, Technical University of Denmark, 2800 Kgs Lyngby, Denmark \\ ${ }^{\mathrm{c}}$ NIL Technology, Diplomvej 81, 2800 Kgs Lyngby, Denmark
}

\begin{abstract}
An optical two-beam trap, composed from two counter propagating laser beams, is an interesting setup due to the ability of the system to trap, hold, and stretch soft biological objects like vesicles or single cells. Because of this functionality, the system was also named "the optical stretcher" by Jochen Guck, Josep Käs and co-workers some 15 years ago. In a favorable setup, the two opposing laser beams meet with equal intensities in the middle of a fluidic channel in which cells may flow past, be trapped, stretched, and allowed to move on, giving the promise of a high throughput device. Yet, single beam optical traps, aka optical tweezers, by far outnumber the existing optical stretchers in research labs throughout the world. The ability to easily construct an optical stretcher setup in a low-cost material would possibly imply more frequent use of the optical stretching technique. Here, we will outline the design, the production procedures, and results obtained in a fiber-based experimental setup built within an injection molded microfluidic polymer chip. The microfluidic chip is constructed with a three layer technology in which we ensure both horizontal and vertical focusing of the cells we wish to trap, thereby preventing too many cells to flow below the line of focus of the two counter propagating laser beams that are positioned perpendicular to the direction of flow of the cells. Results will be compared to that from other designs from previous work in the group.
\end{abstract}

Keywords: optical trapping, fiber-based optical trap, polymer injection molding, hydrodynamic focusing.

*Kirstine Berg-Sørensen, kirstine.berg-sorensen@ fysik.dtu.dk

\section{Introduction}

An optical stretcher setup, most successfully combined with microfluidics, ${ }^{1-5}$ is a versatile tool for measurement of the elastic properties of a single cell, often expressed as the optical deformability of the cell in question. The optical deformability has been demonstrated as a signature of cancer and of metastatic potential, ${ }^{6,7}$ and the optical stretcher has also been employed in investigations of mechanical properties of stem cells. ${ }^{8}$ Different strategies for the actual design of an optical stretcher experiment have been presented in the literature, ranging from the very simple "open" setup in which optical fibers are aligned against e.g a glass capillary, ${ }^{1}$ to microfluidic versions that are more apt for high throughput investigations. This includes systems using commercial optical fibers, for example a setup based on a commercial square capillary in glass as the microfluidic channel, positioned in SU-8 structures modified by standard photolithography that at the same time ensures fiber-alignment, ${ }^{2}$ designs made in polymer materials ${ }^{3}$ and also designs in which the optical field is complemented by a sound wave. ${ }^{9}$ An alternative strategy is to couple laser light into waveguides that may be written in silica by femto-second laser pulses ${ }^{4,10}$ or in polymethylmethacrylate (PMMA). ${ }^{11}$ The silica device also has included the addition of a sound wave to preposition the cells to trap. ${ }^{12,13}$ The benefit of a solution based on waveguides is an inbuilt alignment of the two counterpropagating light beams.

Yet, in the biophysics community, optical trapping in most cases involve the single beam optical trap obtained by a strongly focused Gaussian beam, aka optical tweezers, as first suggested by Arthur Ashkin. ${ }^{14}$ With standardized experimental setups for optical stretchers, based on low-cost polymer microfluidic systems, we believe, however, that optical stretchers may also be more widely

Optical Trapping and Optical Micromanipulation XIII, edited by Kishan Dholakia, Gabriel C. Spalding, Proc. of SPIE Vol. 9922, 992213 - (c) 2016 SPIE · CCC code: 0277-786X/16/\$18 · doi: 10.1117/12.2236465 
used. This paper describes in detail our most recent attempt to design, injection mold and assemble such a system. We will discuss the capability of this latest optofluidic system to study mechanical properties of single cells subject to different environments through demonstrations of the ability of the system to allow for an exchange of the fluid surrounding a trapped particle.

\section{Materials and methods}

The optical trapping experiments were performed with a customized setup, centered around an inverted optical microscope (Leica DMI3000B) equipped with a CCD camera (Thorlabs DFC420M), and a microfluidic polymer system (Cyclic Olefin Copolymer TOPAS polymer 5013) to be described in detail below. The microfluidic chip has two single-mode fibers (AFM Technologies FOP-64-L-5-H64-22) connected via standard fiber connectors to two $1064 \mathrm{~nm}$ fiber-coupled diode lasers (Lumics LU1064M450-1006N10A), a four-channel Fluigent micropump (MFCS-EZ, 0-65 mbar) and custom-written LabVIEW software. The pressure driven pump was connected to the microfluidic system via silicone tubes of diameter $1.5 \mathrm{~mm}$ and around $50 \mathrm{~cm}$ of length (VWR ML94231A). All the experiments presented in this work were performed with $5 \mu \mathrm{m}$ spherical polystyrene microparticles (Fluka; 79633; 10\% solid wt content) mixed in water at 1:4 volume ratio.

\subsection{Design considerations}

\subsubsection{Design of the chip}

The microfluidic system is based on two parts: the top part (lid B) contains inlets/outlet ports with dimensions of standard LUER fittings, while the bottom part of the device (lid A) contains the microchannels (Figure 1). The top part is based on a standard configuration developed for polymer injection molding of microfluidic lab-on-chip systems, ${ }^{15}$ whereas the outline of the channels and fiber grooves in the bottom part was based on our experiences from a previous design. ${ }^{16}$

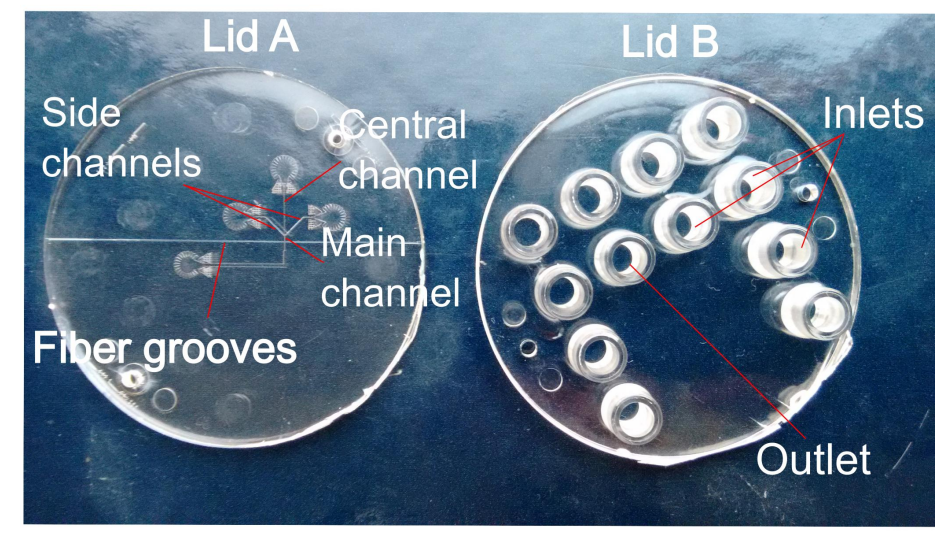

Fig 1 Microchip composed by two part: lid A and lid B. Lid A contains three inlets: Two side channels and a central channel that merge to form one main liquid channel. The fiber grooves are located perpendicular to this main channel. Lid B contains inlets/outlet ports. After mounting the fibers in lid A, the two parts are assembled to form a tightly sealed microfluidic system easily connected to a pumping system through the LUER connectors in lid B. 
To ensure horisontal hydrodynamic focusing, a three inlets structure was chosen. This ensures that cells flow mainly in the center of a main liquid channel. In the vertical direction, a shallower inlet channel assists in vertical positioning of the particles at the focus line of the two laser beams (Figure 2a), oriented perpendicular to the direction of the flow of the particles. Thus, ideally, when the three inlet-streams are mixed, as long as the flow velocity field reaches a reasonable minimum flow speed, cells or particles will partially sediment to preferentially flow through this focus line, with equal distance from the two fiber ends (Figure 2b). At the flow speeds and viscosity similar to that of water applicable here, the contribution from lift forces is at max of order a few per cent, ${ }^{17}$ and our estimate for the good flow speed is based on simple considerations of the force of bouyancy (see appendix for details). For $5 \mu \mathrm{m}$ diameter polystyrene particles, this velocity is estimated to be $4.2 \mu \mathrm{m} / \mathrm{s}$, corresponding to $4.2210^{-5} \mu \ell / \mathrm{s}$.

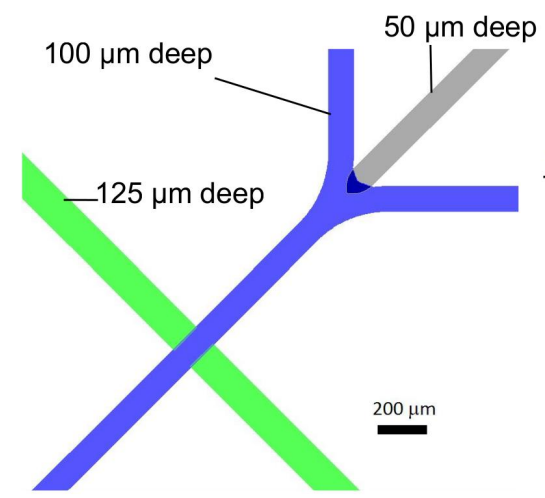

(a)

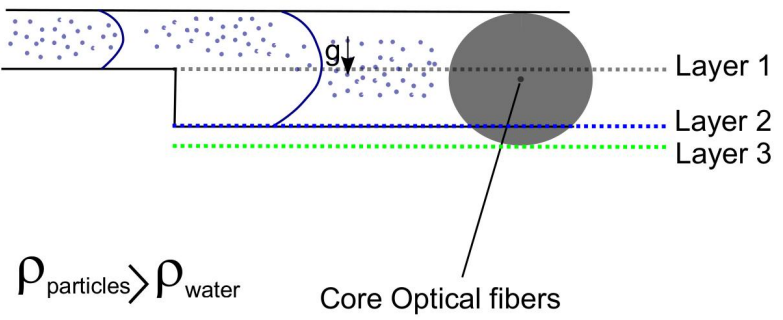

(b)

Fig 2 Hydrodynamic focusing in a three layer technology microchip (a) Top view of the layers channel. A central channel (grey) is located on a top layer with respect the two side and main channels (blue). Fiber grooves are located on a third layer (green) (b) Lateral view of the layers in the chip. Particles or cells flowing in the central channel located at the top, i.e., at and above layer 1, are directed by a combination of partial sedimentation by gravity and fluidic lift into the main channel, i.e., at and above layer 2, to the focus line of the two optical fibers positioned in the grooves perpendicular to the main channel; fibers grooves are at and above layer 3 .

The inlet/outlet ports in lid B include the two side channels of a nominal cross-section of $100 \mu \mathrm{m} \times 100 \mu \mathrm{m}$ with $3250 \mu \mathrm{m}$ of length each before the merging point, and a central channel that is $100 \mu \mathrm{m}$ wide and $50 \mu \mathrm{m}$ deep, $4350 \mu \mathrm{m}$ long before the point in which they merge. The three channels merge into a main channel with a nominal square cross-section of $100 \mu \mathrm{m} \times 100 \mu \mathrm{m}$, $1400 \mu \mathrm{m}$ long (Figure 2a). In the middle of the main channel, at around $620 \mu \mathrm{m}$ from the point where the three channels meet, two grooves of nominal cross-section of $125 \mu \mathrm{m} \times 125 \mu \mathrm{m}$ for housing the optical fibers cross the main channel. Thus, two optical fibers of $125 \mu \mathrm{m}$ diameter may be positioned inside the grooves, preferably with an approximate distance between the end of the fibers of $100 \mu \mathrm{m}$. Near the outlet, the main liquid channel is wider, to reduce hydraulic resistance.

As argued in earlier work, the square geometry of the fiber grooves is well suited for alignment of fibers, reducing their later movement, reducing leaking and favouring fiber gluing. ${ }^{16}$ 


\subsubsection{Injection molded chips}

The injection molded chips were produced based on a multilayer stamp (shim) for injection molding, fabricated with standard cleanroom techniques. ${ }^{18-20}$ The first step is to fabricate the Silicon master. The silicon master for lid A, Fig. 1, was produced by standard UV lithography and deep reactive ion etching of a $150 \mathrm{~mm}$ diameter silicon wafer. The lid A channel layout with three channel depths were made with three UV, $365 \mathrm{~nm}$ wavelength, lithography steps performed on a Sss MA/BA6 UV contact aligner followed by deep reactive ion etching with an STS Bosch process tool, Advanced Silicon Etcher STS MESC Multiplex ICP. The channel layout and depths are shown in Fig. 2. The next step is the fabrication of the Nickel shim. The Nickel shim for injection molding of lid A was made by direct nickel plating on the silicon master. A $90 \mathrm{~nm}$ thick nickel vanadium, NiV, layer was sputtered onto the master surface with a Lesker sputtering tool. Subsequently, the nickel was plated on top of the seed layer in a Technotrans microform.200 nickel electroplating tool. The thickness of the final nickel shim was $330 \mu \mathrm{m}$. Finally, the nickel shim was trimmed into the right format for the injection molding tool by laser cutting while protecting the nickel shim surface with photoresist. The photoresist was cleaned off the nickel shim prior to injection molding. The final step is the injection molding step. Both lid A and lid B parts were injection molded in the biocompatible cycloolefin copolymer Topas 5013L-10 (Topas Advanced Polymers, US). Topas pellets were pre-dried in order to ensure optimal rheological performance during injection molding, which was done on a modified Engel Victory 80/45 Tech, that allows for applying variothermal processes. The maximum tool clamping force on the specific machine is $450 \mathrm{kN}$. To ensure an excellent pattern replication fidelity during injection molding, both a conventional isothermal and a variothermal process were applied: For Lid B, the nickel shim was kept at $120^{\circ} \mathrm{C}$ (i.e. slightly above the glass transition temperature, $T_{g}$, of the Topas) during the cavity filling injection step, whereas for Lid A, the variothermal process was used, starting at $145^{\circ} \mathrm{C}$ and rapidly cooling down to under $135^{\circ} \mathrm{C}$. The variothermal process allows us to get the best possible filling without defects on the edges that would otherwise hamper the subsequent bonding. In both cases, the injection step lasts around one and a half minute. The polymer melt temperature at the nozzle was set to $250^{\circ} \mathrm{C}$ and a holding pressure of 1150 bar was applied during the subsequent cooling below $T_{g}$.

\subsubsection{Mounting the fibers and bonding the chip}

After the chip fabrication, two optical fibers were prepared and inserted into the grooves (lid A). Different procedures for bonding the chip has been investigated, simple tape-bonding by doublesticky tape and UV assisted thermal bonding, the latter is described here. In order to prevent misalignment during the bonding process, two small drops of Epoxy glue (Casco 2806) were adhered to the fiber grooves on top of the fiber, $2 \mathrm{~mm}$ from the main liquid channel. As a next step, lid B was chemically activated by placing it in UVI light for 2 minutes and then rapidly positioned on the top of lid A by carefully matching the inlets of lid A with the ports in lid B. UV assisted thermal bonding is performed in a hydraulic press (P/O/Weber, Germany) at $125^{\circ} \mathrm{C}$ and 5 bar after both polymer parts are exposed to a 400W mercury lamp (Dymax). The glass transition of TOPAS 5013 is $130{ }^{\circ} \mathrm{C} .{ }^{21}$ The coefficient of linear thermal expansion of TOPAS polymer is around $0.610^{-4} K^{-1}$ whereby heating from room temperature to $125^{\circ} \mathrm{C}$ theoretically results in fiber ends being displaced by up to $20 \mu \mathrm{m}$ along the fiber grooves. 
In order to reduce the polymer expansion and therefore the misplacement of the optical fibers, the microchip was placed in the pressure bonding machine while the pressure plates were still cold. After that, first 5 bar pressure was applied and secondly, the temperature was set to $125^{\circ} \mathrm{C}$. When the system reached $125^{\circ} \mathrm{C}$, the chip was left in the bonding press for approximately 10 minutes.

\section{Characterization of Si master, shim and microfluidic chips}

The Si-master, the shim and the final microfluidic chips are inspected by optical microscopy and channel depths in the Si-master are measured by mechanical profilometry. The latter provided depths of the three layers of $49.5 \mu \mathrm{m}, 99.7 \mu \mathrm{m}$ and $123.5 \mu \mathrm{m}$ respectively. Microscopy images of the Si-master and the shim are shown in Fig. 3.

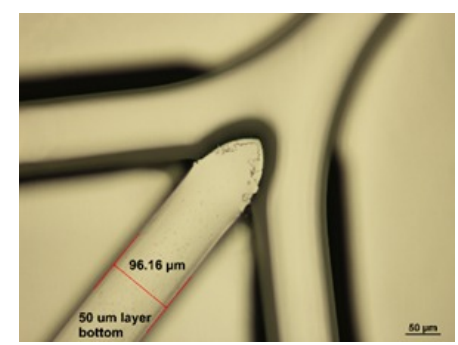

(a)

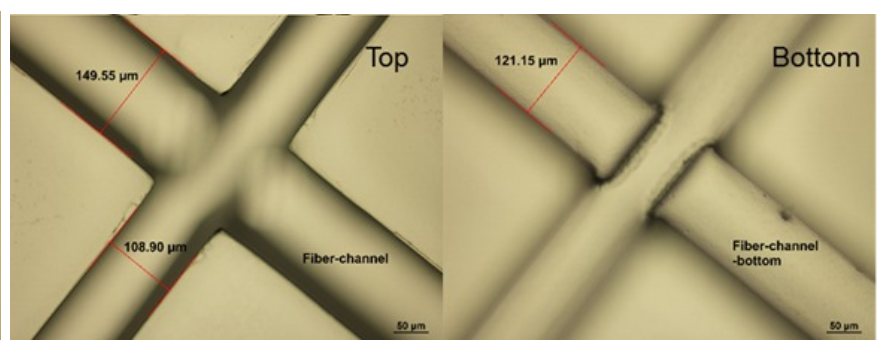

(b) (c)

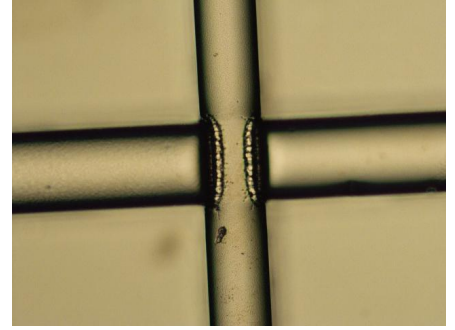

(d)

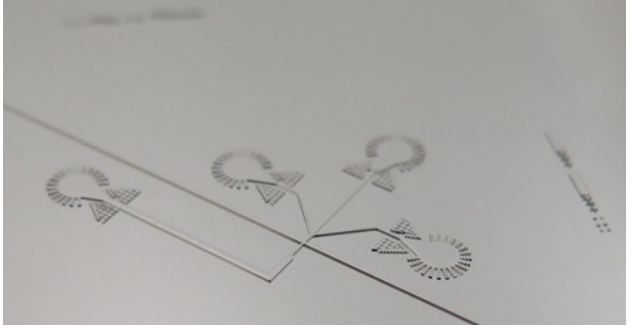

(e)

Fig 3 Optical microscopy images of the Si-master, and the shim. Part (a) shows the Si-master at the position where the three channels of the inlet structure meets, and at the top (b) and bottom (c) of the position where the main liquid channel crosses the fiber grooves. Part (d) is an image of the Ni-shim, focused at the bottom of the liquid channel, and illustrates that the etch-marks in the Si-master, visible in part (c), are carried over to the shim and will also be visible in the final injection molded chips. Panel (e) shows a close-up photograph of the Ni-shim.

\section{Results: Optical trapping experiments}

\subsection{Improved trapping efficiency}

Video 4 shows the trapping of several particles, each at different time, in the space between the two optical beams positioned perpendicular to the direction of the flow. As can be seen in the plane view, in several instants a high fraction of the particles flows in the focus plane of laser beam, i.e. 4 particles out of 5 at 00:00:02. The high fraction of focused particles above the laser beam line allowed to efficiently trap them at high rate, up to 15 particles in 25 seconds. Additionally, the precise alignment of the fibers resulted in a highly focused optical power, i.e. to the center of the main channel for an efficient trapping of particles. 


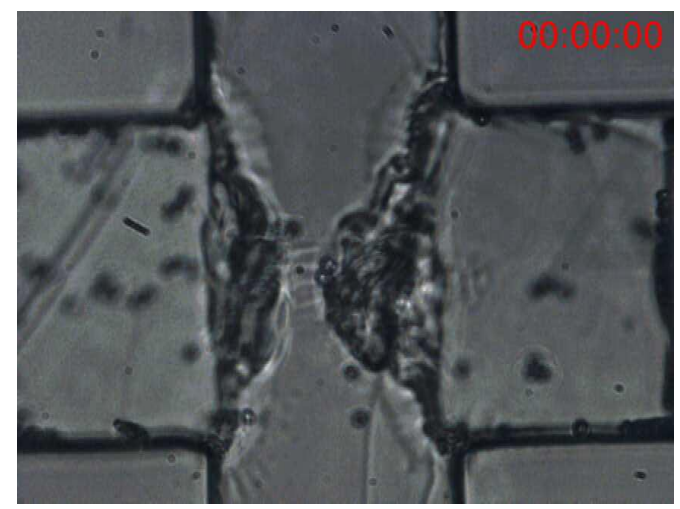

Video 4 Trapping of several particles in the main channel between two optical beams possitioned perpendicular to the direction of the flow. The debris in front of the fiber that blurs the image was unfortunately impossible to remove, yet we preferred to use this chip as fiber ends are positioned particularly close, as will be discussed further below. http://dx.doi.org/10.1117/12.2236465

\subsection{Fluid exchange while a bead is trapped}

Figure 5 displays a time series during the process of fluid exchange while a particle is trapped.

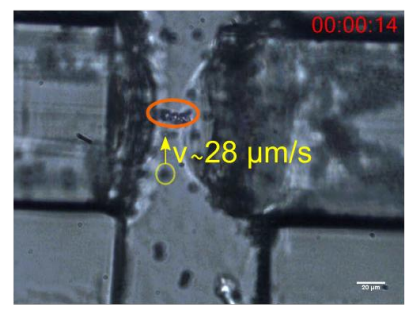

(a)

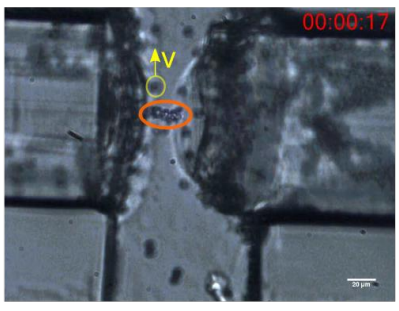

(b)

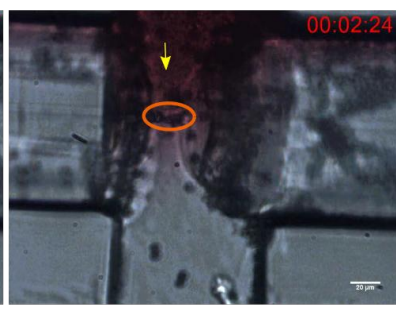

(c)

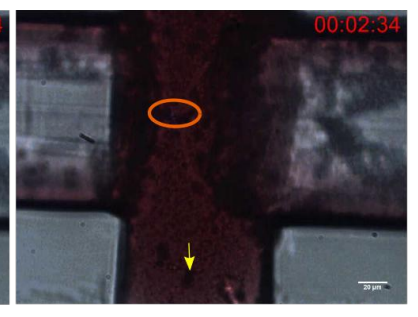

(d)

Fig 5 Time series during the process of exchanging the fluid while three particle are trapped. In (a) and (b) three particles are trapped (orange) in the main channel, between the two optical fibers. In the background, a yellow arrow indicates the direction and velocity of unfocused particles flowing in a laminar flow with a velocity $28 \mu \mathrm{m} / \mathrm{s}$ (c) The direction of the fluid is changed (yellow arrow) visualizing the entrance of a red dye to the space where the particles are still trapped (orange). (d) The liquid in the environment of the trapped particles has been complety exchanged.

Figs. 5a and 5b show three trapped particles in a flow with an approximate velocity of $28 \mu \mathrm{m} / \mathrm{s}$. Notice that in order to exchange the fluid around a trapped particle, the flow direction needs to be inverted (Fig 5c). Therefore, the pressure at the outlet was gradually decreased to zero followed by an increase of the pressure in the central inlet and a somewhat lower pressure in the two side channels. In order to better visualize changes of the direction of the fluid and also the exchange of liquid around the trapped particles, a red dye was used. Fig. 5c visualizes the entrance of the red color dye in the place where the particles are still trapped and in Fig. 5d, the liquid in the environment of the trapped particles has been completely exchanged. 


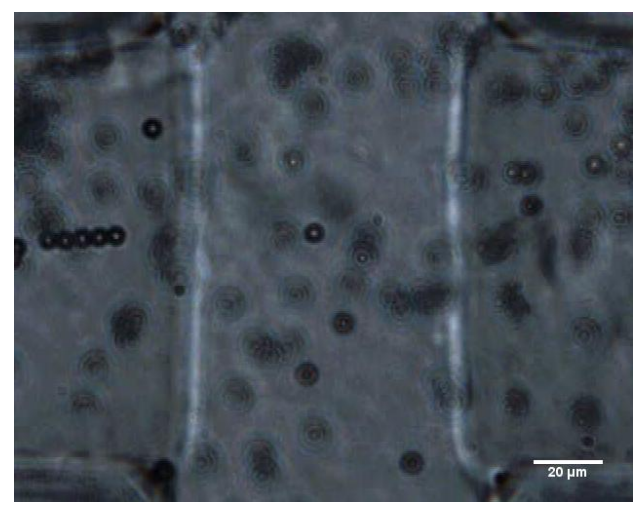

Video 6 First generation microfluidics-devices from previous work. ${ }^{16}$ Trapping of several particles in the main channel between two optical beams possitioned perpendicular to the direction of the flow. In the background, a high fraction of particles flowing out of the focus line of the two laser beams are visible. http://dx.doi.org/10.1117/12.2236465

\section{Discussion and conclusion}

\subsection{Comparison with other devices}

The new design of a polymer optofluidic device shows clear advantages compared with the former generation. ${ }^{16}$

First, the short length of the main channel compared with the first generation chip implies that the pressure drop at the desired flow speed is lower. During the experiments we found that this fact facilitated a precise control of flow speed and flow direction in the channel. Thereby, experiments requiring exchange of the liquid surrounding a trapped cell become more feasible, opening new capabilities for this optical trapping setup for further applications to investigate cell responses.

Second, the three layer technology that assists hydrodynamic focusing ensures that a high fraction of particles flow in the focus plane of the counter propagating laser beams, thus allowing to trap 15 cells in 25 seconds. As comparison, in the first generation microfluidics-devices of reference 16, an experiment with fiber ends deliberately placed equally far apart for direct comparison, results in a fraction of around 5/60 flowing in the focus plane (video 6).

Due to the mechanical traction applied during the sealing of the chip, we observed, however, the final distance between the ends of the two optical fibers as large as $300 \mu \mathrm{m}$. This deformation could be explained by a possible surface modification of lid A during the bonding procedure. When sealing the chip, lid A expands thermally, and through the combined action of thermal expansion and applied pressure, lid A may sink into the luer cavities of lid B causing also a deformation of the fiber grooves. In the previous generation device, the injection molded part of the chip contained both liquid channels, fiber grooves and LUER ports, and the chip was sealed either thermally with a thin disk or, even simpler, by pressure bonding a foil prepared with pressure assisted glue. In spite of this undesired movement of the fibers, the preliminary results presented here with polystyrene beads indicate the capability of the chip to trap a higher fraction of cells, compared with the device discussed in reference 16.

Compared to devices in which alignment of the laser beams is ensured by either direct laser writing of waveguides in an all glass device, ${ }^{4,10,12}$ or by DUV writing in a polymer device, ${ }^{9}$ delivery of light by optical fibers enables a high laser intensity for trapping and stretching even with, say, a fiber-coupled diode laser of relatively low power (200-500 mW). Laser written or DUV written 
waveguides have, compared to the optical fiber, a high loss, both from propagation losses - the reported values are $0.66 \mathrm{~dB} / \mathrm{mm}$ in the polymer and $0.9 \mathrm{~dB} / \mathrm{cm}$ in the glass chips - and from coupling losses.

In earlier work, we have also investigated the applicability of sound waves to ensure that cells and beads to trap flow at the height of the focus line between the two trapping laser beams, ${ }^{9,12,13}$ so-called acoustophoretic prefocusing. With acoustophoretic prefocusing, one can thus avoid the situation often encountered with optical stretcher devices that each new cell type requires a different position of the counter propagating light beams with respect to the floor of the liquid channel. ${ }^{2}$ The ability to prefocus the cells is therefore a clear advantage. Unfortunately, material parameters of polymers that may be injection molded are not well suited for acoustophoresis, ${ }^{22}$ and therefore hydrodynamic focusing, both in the horisontal and the vertical direction, was explored in the chip design discussed here.

\subsection{Conclusion and outlook}

As demonstrated by the microfluidic chip discussed in this manuscript, as well as the microfluidic system we presented earlier, in reference 16, microfluidic chips of polymer materials, and produced by production ready methods, are readily available for assembly of optical stretcher devices. The assembly still relies on post-processing steps, and there is still work to do to optimize the procedures that ensure a tight sealing and thereby ensures chips that work similarly for each device prepared.

Nevertheless, the preliminary results presented in the manuscript illustrates that the microfluidic system is available for trapping and stretching experiments in which liquid exchange may change the local environment of the trapped cell. Thereby the stretching response of the same cell, subject to different liquids, may be investigated. We imagine for example experiments that investigate membrane-curvature dependence of membrane-proteins in a trapped cell.

\section{Appendix: Sedimentation-assisted vertical positioning}

The sedimentation-assisted positioning may be estimated based on standard equations for Stokes drag. For particles of density $\rho_{\text {bead }}$ and radius $r$, a simple estimate of the required flow speed $v_{x}$ along the flow channel for hydrodynamic focusing to the position of the fiber cores, is based on the sedimentation time over the distance $h$ in the vertical $z$-direction.

$$
v_{x}=\frac{s_{x}}{t}, t=\frac{h}{v_{z}}, \text { and } v_{z}=\frac{4 \pi r^{3}}{3}\left(\rho_{\text {bead }}-\rho_{\text {fluid }}\right) g \frac{1}{6 \pi \eta r} \equiv \frac{F_{\text {sed }}}{6 \pi \eta r} \text {. }
$$

Here, $\rho_{\text {fluid }}$ and $\eta$ are the density and viscosity of the fluid and $g$ is the acceleration of gravity. For $s_{x}$ we use the distance between the merging point of the three inlet channels and the fiber grooves, $s_{x} \simeq 620 \mu \mathrm{m}$, and for $h$ the distance from the top of the microfluidic channel to the position of the fiber cores, $h \simeq 62 \mu \mathrm{m}$. We also introduced the notation $F_{\text {sed }}$ for the sedimentation force.

In order to estimate the corresponding lift force, we apply the result derived by Saffman, ${ }^{17} F_{\text {lift }}$,

$$
F_{\text {lift }} \simeq 6.46 \sqrt{\eta \rho_{\text {fluid }}} v_{0} r^{2} \sqrt{\left|\frac{\partial v_{x}}{\partial z}\right|},
$$


where $v_{0}$ is the relative velocity between particle center and surrounding fluid. The derivative $\partial v_{x} / \partial z$ may be estimated from the standard expression for Poisseuille flow in a circular tube of radius $R$ and length $\ell$,

$$
v_{x}(y, z)=\frac{\Delta p}{4 \eta \ell}\left(R^{2}-y^{2}-z^{2}\right) ; \frac{\partial v_{x}}{\partial z}=-\frac{\Delta p}{2 \eta \ell} z
$$

giving an overestimate for the order of magnitude for the derivative of $\left|\partial v_{x} / \partial z\right| \sim \Delta p R /(2 \eta \ell)$. Similarly, for an order of magnitude estimate, we use a typical pressure-drop of order $0.2 \mathrm{mbar}, \ell$ of order $4000 \mu \mathrm{m}, R$ of $50 \mu \mathrm{m}, r$ of $2.5 \mu \mathrm{m}$, and the density and viscosity of water. For $v_{0}$, the value of $v_{x}$ in Eq. (1) provides an overestimate. This allows us to evaluate the ratio of lift force to sedimentation force

$$
\frac{F_{\text {lift }}}{F_{\text {sed }}} \simeq \frac{6.46}{6 \pi} \frac{r s_{x}}{\eta h} \sqrt{\frac{\Delta p \rho_{\text {fluid }} R}{2 \ell}}
$$

which provides an overestimate of the ratio between lift and sedimentation of 9.6 per cent.

\section{Acknowledgments}

We are grateful to the support provided by the PolyNano network, allowing for the development and production of the microfluidic chip.

\section{References}

1 J. Guck, R. Ananthakrishnan, H. Mahmood, et al., "The optical stretcher: A novel laser tool to micromanipulate cells," Biophys. J. 81(2), 767 - 784 (2001).

2 B. Lincoln, S. Schinkinger, K. Travis, et al., "Reconfigurable microfluidic integration of a dual-beam laser trap with biomedical applications," Biomed. Microdevices (2007).

3 F. Lautenschlaeger and J. R. Guck, "Microfluidic integration of high power dual-beam laser traps for cell mechanical measurements," in IEEE ISOT: 2009 International Symposium on Optomechatronic Technologies, 419-422 (2009).

4 N. Bellini, K. Vishnubhatla, F. Bragheri, et al., "Femtosecond laser fabricated monolithic chip for optical trapping and stretching of single cells," Opt. Express 18(5), 4679-4688 (2010).

5 T. Yang, F. Bragheri, and P. Minzioni, "Review: A comprehensive review of optical stretcher for cell mechanical characterization at single-cell level," Micromachines 7, 90 (2016).

6 J. Guck, S. Schinkinger, B. Lincoln, et al., "Optical deformability as an inherent cell marker for testing malignant transformation and metastatic competence," Biophys. J. 88, 3689-3698 (2005).

7 T. W. Remmerbach, F. Wottawah, J. Dietrich, et al., "Oral cancer diagnosis by mechanical phenotyping," Cancer. Res. 69, 1728-1732 (2009).

8 A. E. Ekpenyong, G. Whyte, K. Chalut, et al., "Viscoelastic properties of differentiating blood cells are fate- and function-dependent," PLOS ONE 7 (2012).

9 M. Khoury, R. Barnkob, L. L. Busk, et al., "Optical stretching on chip with acoustophoretic prefocusing," Proc. SPIE 8458, 84581E (2012).

10 N. Bellini, F. Bragheri, I. Cristiani, et al., "Validation and perspectives of a femtosecond laser fabricated monolithic optical stretcher," Biomed. Opt. Express 3(10), 2658-2668 (2012). 
11 M. Khoury, C. Vannahme, K. T. Sørensen, et al., "Monolithic integration of DUV-induced waveguides into plastic microfluidic chip for optical manipulation," Microelectron. Eng. 121, 5-9 (2014).

12 G. Nava, F. Bragheri, T. Yang, et al., "All-silica microfluidic optical stretcher with acoustophoretic prefocusing," Microfluid. Nanofluid. 19, 837-844 (2015).

13 T. Yang, F. Bragheri, G. Nava, et al., "A comprehensive strategy for the analysis of acoustic compressibility and optical deformability on single cells," Scientific Reports 6, 23946 (2016).

14 A. Ashkin and J. Dziedzic, "Optical trapping and manipulation of viruses and bacteria," Science 235, 1517-1520 (1987).

15 "PolyNano - strategic research center in precision and nano-scale polymer mass replication of biocchips; www.polynano.org."

16 M. Matteucci, M. Triches, G. Nava, et al., "Fiber-based, injection-molded optofluidic systems: Improvements in assembly and applications," Micromachines 6, 1971-1983 (2015).

17 P. G. Saffman, "The lift on a small sphere in a slow shear flow," J. Fluid Mech. 22, 385-400 (1965).

18 P. Utko, F. Persson, A. Kristensen, et al., "Injection molded nanofluidic chips: Fabrication method and functional tests using single-molecule dna experiments," Lab on Chip 11, 303308 (2011).

19 S. Tanzi, P. F. Østergaard, M. Matteucci, et al., "Fabrication of combined-scale nano- and microfluidic polymer systems using a multilevel dry etching, electroplating and molding process," J. Micromech. Microeng. 22, 115008 (2012).

20 M. Matteucci, T. L. Christiansen, S. Tanzi, et al., "Fabrication and characterization of injection molded multi level nano and microfluidic systems," Microelec. Eng. 111, 294-298 (2013).

21 "Topas advanced polymers, cyclic olefin copolymer (coc); http://www.topas.com/company/news/topas-advanced-polymers-cyclic-olefin-copolymercoc-elastomer-earns-fda-food-contact."

22 H. Bruus, Theoretical Microfluidics, Oxford University Press, New York (2008). 